\title{
N-Oxides of Adenosine-Type Nucleosides Undergo Pyrimidine Ring Opening and Closure To Give 5-Amino-4-(1,2,4-oxadiazol-3-yl)imidazole Derivatives
}

\author{
Ireneusz Nowak, John F. Cannon, and Morris J. Robins ${ }^{*}$ \\ Department of Chemistry and Biochemistry, Brigham Young University, Provo, Utah 84602-5700 \\ morris_robins@byu.edu
}

Supporting Information (3)

Pages S36-S38: X-Ray crystal structures of $\mathbf{3 c}, \mathbf{1 1 b}$, and 12a.

Pages S39-: X-Ray crystallographic data in CIF format for $\mathbf{3 c}, \mathbf{1 1 b}$, and $\mathbf{1 2 a}$. 


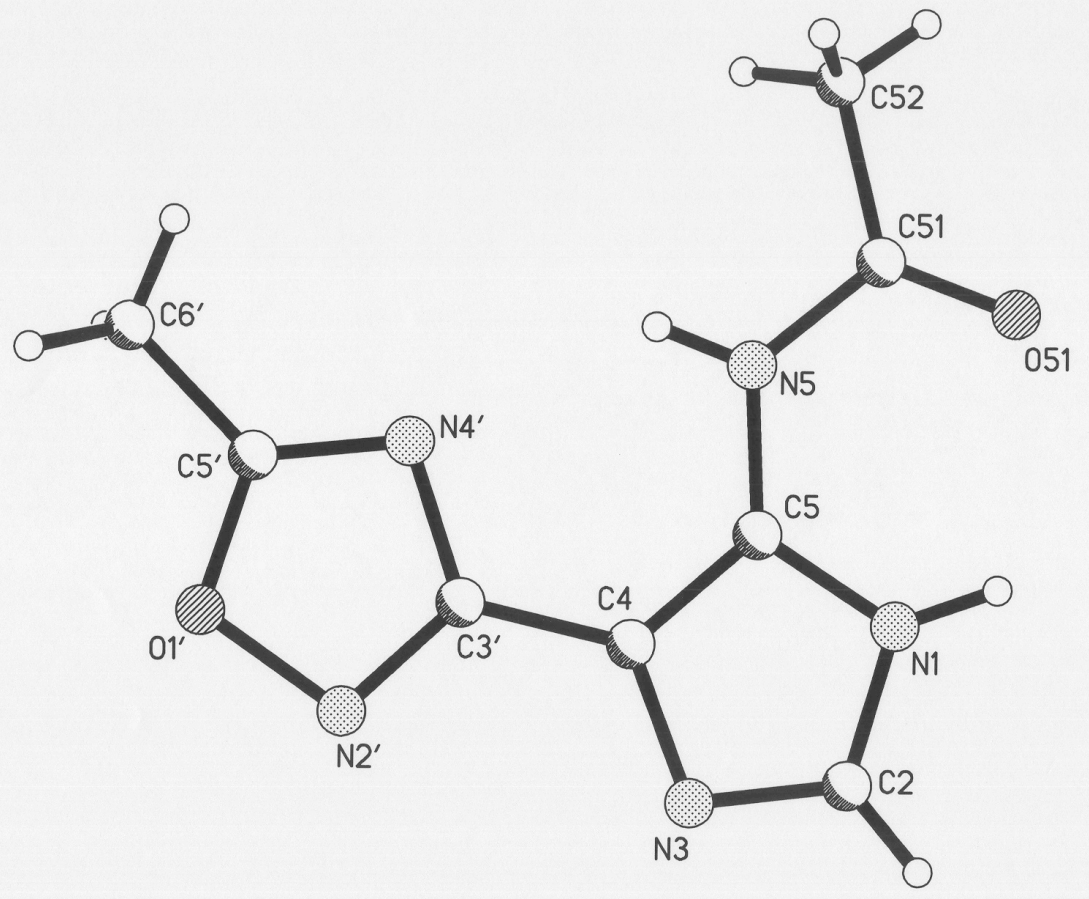

X-Ray crystal structure of compound 3c (coded XL1031). 


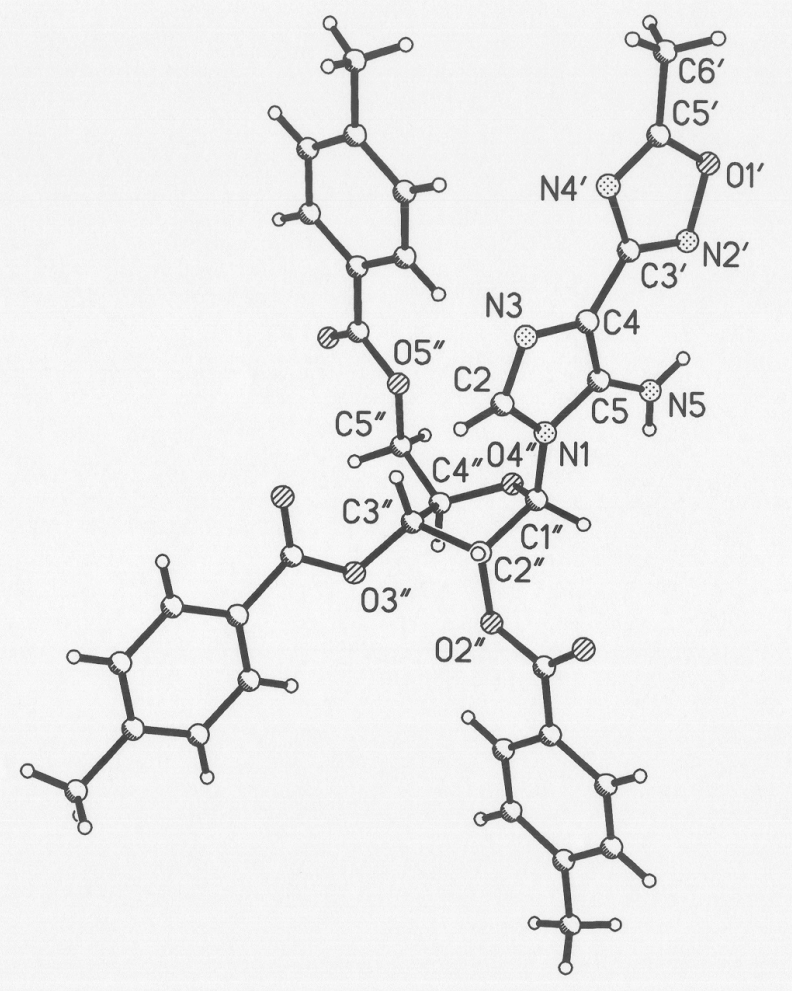

X-Ray crystal structure of compound 11b (coded XL1012). 


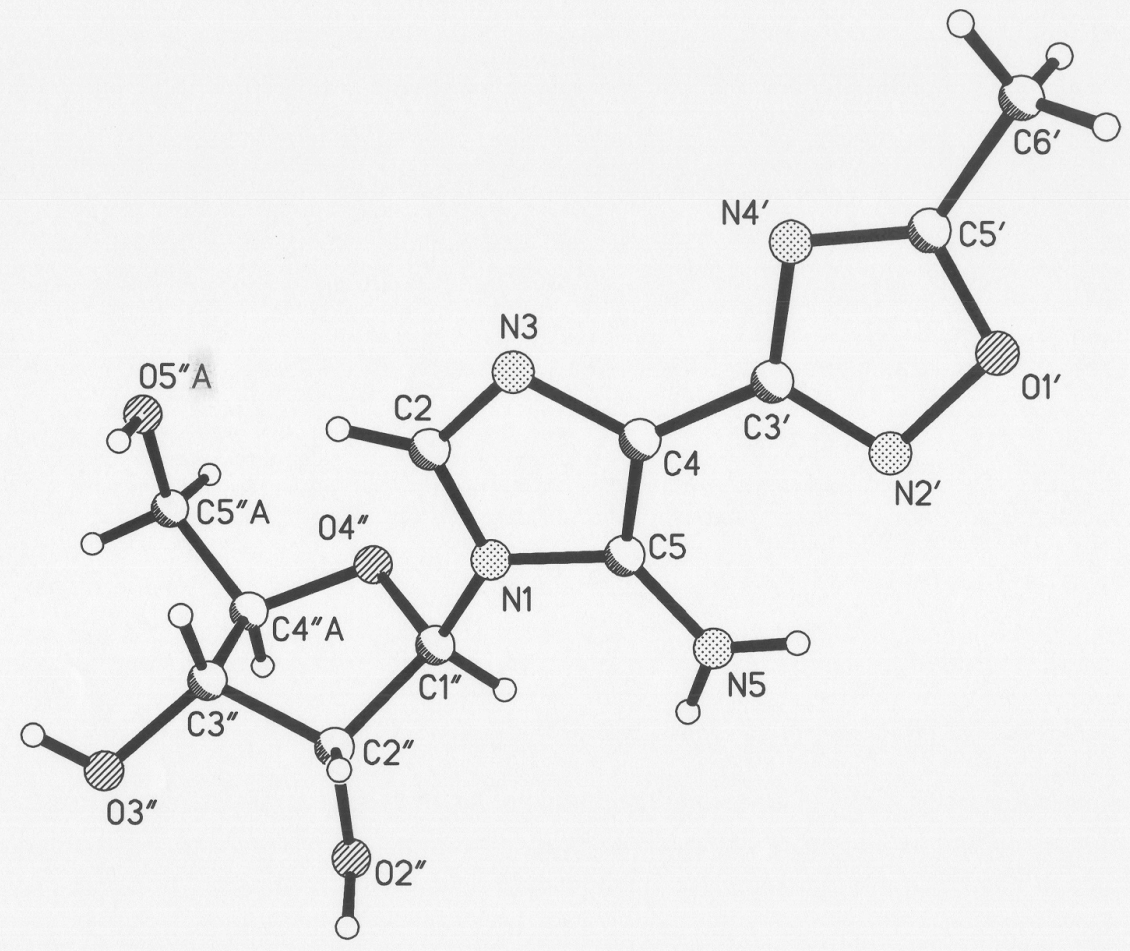

X-Ray crystal structure of compound 12a (coded XL904). 
